\title{
Force Measurements of Single and Double Barrier DBD Plasma Actuators in Quiescent Air
}

\author{
Alan R. Hoskinson* and Noah Hershkowitz ${ }^{\dagger}$ \\ University of Wisconsin-Madison \\ David E. Ashpis ${ }^{\ddagger}$ \\ NASA Glenn Research Center at Lewis Field, Cleveland, Ohio, USA
}

\begin{abstract}
We have performed measurements of the force induced by both single (one electrode insulated) and double (both electrodes insulated) dielectric barrier discharge plasma actuators in quiescent air. We have shown that, for single barrier actuators, as the electrode diameter decreased below those values previously studied the induced force increases exponentially rather than linearly. This behavior has been experimentally verified using two different measurement techniques: stagnation probe measurements of the induced flow velocity and direct measurement of the force using an electronic balance. In addition, we have shown the the induced force is independent of the material used for the exposed electrode. The same techniques have shown that the induced force of a double barrier actuator increases with decreasing narrow electrode diameter.
\end{abstract}

\section{Introduction}

Numerous groups have demonstrated that dielectric barrier discharges (DBDs) generating plasma on a flat surface using an asymmetric electrode configuration can induce a flow of neutral gas when operated in ambient air. ${ }^{1-5}$ This effect is believed to be similar to the "electric wind" phenomenon noted in corona discharges, ${ }^{6}$ caused by the collision of field-accelerated plasma particles with the neutral gas. When the discharge is used for active flow control, it is commonly termed a plasma actuator.

Groups led by Roth,$^{7}$ Corke, ${ }^{3}$ and others demonstrated via wind tunnel experiments that airfoils with operating plasma actuators could be raised to higher angles of attack without experiencing flow separation than airfoils without an actuator. Single barrier plasma actuators, such as the one diagrammed in figure 1, have been studied intensely both experimentally ${ }^{4,5,8-10}$ and computationally. ${ }^{6,11-13}$

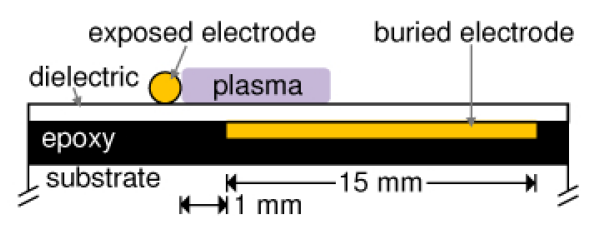

Figure 1. Single barrier actuator

Double barrier actuators, such as the one pictured in figure 2, have been much less widely studied than the single barrier geometry, in part due to the observation of lower induced velocities. ${ }^{14}$ We include them in this study as they reduce possible sources of irregularities in the experiments. Double barrier actuators have

\footnotetext{
*Graduate Research Assistant, Engineering Physics, University of Wisconsin, Madison, Wisconsin 53706, AIAA Student Member

†Irving Langmuir Professor, Engineering Physics, University of Wisconsin, Madison 53706

${ }^{\ddagger}$ National Aeronautics and Space Administration, Glenn Research Center at Lewis Field, Cleveland, Ohio 44135, USA, AIAA Member
} 


\section{AIAA-2009-485 47th ASM, Orlando, FL}

no features above the surface to impede the induced air flow, and the electrodes are not in physical contact with the plasma so their material characteristics should not affect the discharge.

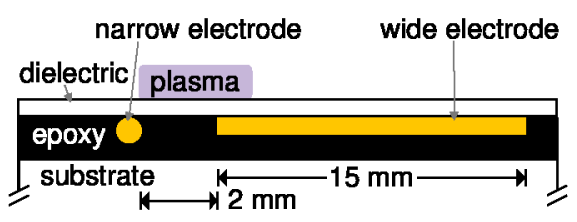

Figure 2. Double barrier actuator

Previously, Enloe et al. ${ }^{4}$ noted that decreasing the diameter of the exposed electrode of a single barrier actuator linearly increased the actuator force without changing the discharge power or emitted light. Increasing the actuator force is highly desirable, but based on the linear extrapolation of Enloe's data only a limited benefit could be obtained by reducing the exposed electrode diameter. In this paper we show that for small diameter electrodes the induced force increases exponentially, rather than linearly, with decreasing diameter. Double barrier actuators show a similar increase in force with decreasing narrow electrode diameter, although this effect is weaker than for single barrier actuators.

Many of the results discussed in this conference paper have been recently published in Hoskinson et al. ${ }^{15}$

\section{Measurement Techniques}

This work used two independent techniques to measure the force induced by each plasma actuator. Data from a stagnation probe approximately determined the velocity distribution near each actuator, which was then converted to the force of the actuator on the air as described below. This technique provided excellent sensitivity for small forces. A more direct measurement of the force, with limited sensitivity, was made with an electronic balance. In addition to the force measurements, the discharge power was determined by integrating the change in charge on a capacitor in the actuator's ground path over an integer number of voltage cycles.

\section{A. Actuator Geometries}

The two actuator geometries considered in this work, single and double barrier, are shown in figures 1 and 2, respectively. The induced air flow moves from left to right in those figures. For all actuators used in this work, the dielectric layer was made from $0.25 \mathrm{~mm}$ thick polyester film, which had a dielectric constant of 3.3. An insulating epoxy filled air gaps beneath the dielectric layer, and had a dielectric constant of 3.8. The substrate layer was used for mechanical mounting, and is not believed to be involved in device performance.

Buried or wide electrodes were made from $40 \mu \mathrm{m}$ thick copper tape, while exposed and narrow electrodes were made from a variety of materials. All electrodes spanned $60 \mathrm{~mm}$ in the direction perpendicular to the plane of figures 1 and 2. For all single barrier actuators a $1 \mathrm{~mm}$ horizontal gap separated the closest edges of the exposed and buried electrodes. For all double barrier actuators the horizontal gap between narrow and wide electrodes was $2 \mathrm{~mm}$. This wider gap was necessary to avoid electric breakdown of the epoxy at higher applied voltages.

For all measurements presented here, the actuators were driven by symmetric triangle waveforms oscillating at $1 \mathrm{kHz}$. The applied voltages were supplied by a Trek 20/20B high voltage amplifier passed through a two-stage low-pass filter to remove RF frequency noise on the signal. In all cases the high voltage was connected to the exposed electrode (for single barrier actuators) or the narrow electrode (for double barrier actuators), with the buried or wide electrodes connected to ground.

\section{B. Stagnation Probe Measurements}

We measured induced air velocities using a small, movable stagnation probe, as shown in figure 3 . To aid in positioning and eliminate ambient air currents, these measurements were conducted in a sealed cylindrical chamber $17 \mathrm{~cm}$ tall and $30 \mathrm{~cm}$ in diameter, with the actuator located near the center of the chamber. The stagnation probe consisted of a glass capillary tube $0.4 \mathrm{~mm}$ in inner diameter with $0.2 \mathrm{~mm}$ thick walls. A 


\section{AIAA-2009-485 47th ASM, Orlando, FL}

Dwyer model 607-01 differential pressure transmitter measured the pressure difference between the probe and a reference port on the bottom of the chamber.

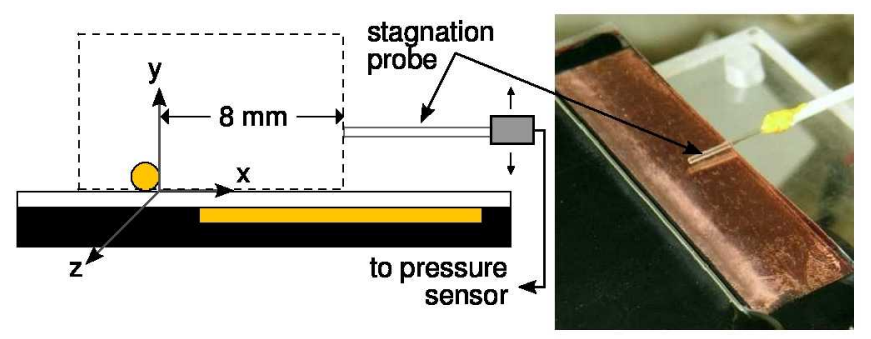

Figure 3. Orientation of stagnation probe for flow measurements and the control volume used in the analysis

For velocity measurements the stagnation probe was pointed into the induced flow: the $-x$ direction as shown in figure 3. All measurements are time-averaged. The measurement technique is similar to that used by Pons et al. ${ }^{9}$

To make comparisons between the stagnation probe and balance measurements, we must convert the measured pressures into forces. The analysis that follows is similar to that performed by Baughn et al., ${ }^{16}$ although our system has no background flow velocity. To analyze the system, we consider a rectangular control volume on the actuator surface which includes the entire plasma volume. The momentum balance equation for the $x$ direction as

$$
\begin{aligned}
\frac{F_{\text {plasma }}}{L_{z}}+\frac{F_{\text {shear }}}{L_{z}}= & \underbrace{\rho \int_{\text {right }} v_{x}^{2} d y}_{\text {I }}+\underbrace{\rho \int_{\text {top }} v_{x} v_{y} d x}_{\text {II }} \\
& -\underbrace{\rho \int_{\text {left }} v_{x}^{2} d y}_{\text {III }}+\underbrace{\int_{\text {right }} p d y}_{\text {IV }}-\underbrace{\int_{\text {left }} p d y}_{\mathrm{V}} .
\end{aligned}
$$

where the integral subscripts indicate over which side of the control volume the integral is performed, and $L_{z}$ is a unit length in the $z$ direction. The included surface forces are static pressures on left and right surfaces and shear acting between the dielectric layer and the air flow. We note that since the shear force acts to slow the $+x$-directed flow, it is always negative. The only included body force is the force of the plasma itself on the gas.

To limit the amount of data required to infer the force of each actuator, we have simplified equation (1) by making several assumptions. The validity and effects of these assumptions are evaluated in section III.C. First, we assume that the static pressure is constant everywhere, and equal to the reference pressure at the chamber bottom:

$$
p=p_{\text {ref }} \text {. }
$$

As the chamber was open to room air prior to being sealed off to reduce measurement noise, $p_{\text {ref }}$ is approximately equal to atmospheric pressure for all measurements. The assumption of constant static pressure allows us to neglect terms IV and V in equation (1), as they will cancel. It also permits a simple conversion from the measured stagnation pressure to a velocity:

$$
v_{x} \approx \sqrt{2\left(p_{0}-p_{\text {ref }}\right) / \rho}
$$

Here $p_{0}$ is the measured stagnation pressure and $\rho$ is the density of air. Previous measurements by Enloe et al. ${ }^{17}$ indicated that air density fluctuations in the vicinity of an actuator are generally smaller than $2 \%$ of the background density. The assumption of a constant static pressure was implicitly made by Pons et al. ${ }^{9}$ in their measurements near a single barrier actuator, as they used an equation like (3) to determine the velocity. In addition, we neglect the momentum flux into the control volume through the left and top edges, setting terms II and III in equation (1) to zero.

Once these assumptions are made, equation (1) simplifies to

$$
\frac{F_{\text {plasma }}}{L_{z}}+\frac{F_{\text {shear }}}{L_{z}} \approx \rho \int_{\text {right }} v_{x}^{2} d y
$$




\section{AIAA-2009-485 47th ASM, Orlando, FL}

In practice, we use a skewed Gaussian function,

$$
v_{\mathrm{fit}}=v_{0} \sqrt{y} \exp \left[-\left(\frac{y-y_{0}}{L}\right)^{2}\right]
$$

fit to the measured data, when evaluating equation (4). An example fit is shown in figure 4.

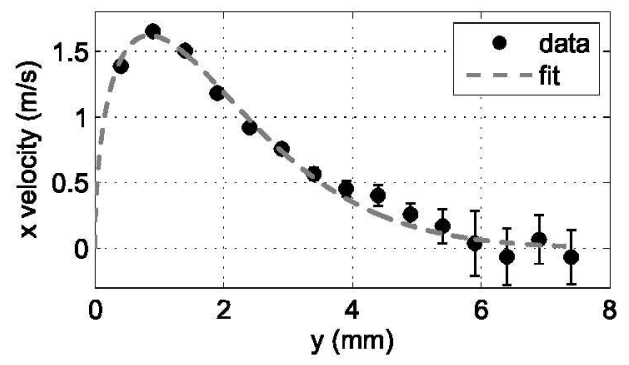

Figure 4. Skewed Gaussian fit to the velocities measured near a single barrier actuator with $d=0.11 \mathrm{~mm}$

This final result is equivalent to the calculations by Baughn et al. ${ }^{16}$ with the background velocity reduced to zero. That study measured the absolute plasma force and so also subtracted a shear force. Here we compare the calculated stagnation probe forces to those measured directly with an electronic balance; since the balance measurements inherently include the shear force, we leave it on the left-hand side of equation (4). All stagnation pressures used for force estimates were made at $x=8 \mathrm{~mm}$. Our calculations therefore only include the shear force up to $8 \mathrm{~mm}$, while the balance measurements include the shear force out to the edge of the dielectric at approximately $16 \mathrm{~mm}$. We estimate the effect of this discrepancy in section III.C.

\section{Electronic Balance Measurements}

To obtain a more direct measurement of the plasma actuator-induced force, we have used an Ohaus model PA313 electronic balance to determine the force. The balance has $1 \mathrm{mg}\left(10^{-5} \mathrm{~N}\right)$ resolution. While this resolution is coarser than that allowed by the stagnation probe measurements, the balance measurement is significantly more direct.

The force measurement was made by mounting the actuators on a stand with the air flows directed upwards. The downward reaction force on the actuator is then measured by the balance as a positive force. Measurements on the electronic balance were conducted inside an acrylic box to prevent room air currents from affecting the measurement.

The high voltage and ground connections were made to the actuator using light 32 AWG wire to eliminate any force caused by sagging wires. Two undesirable effects caused by the high voltage signal were noted and removed. Electromagnetic coupling between the high voltage line and the balance manifested itself as a spurious negative force until the balance was covered with a grounded copper mesh. An insulating platform fit through a hole in the mesh and provided a place on which to set the actuator stand. An electrostatic attraction between the high voltage wire and the copper mesh was minimized by making the actuator stand tall enough to keep the wire approximately $15 \mathrm{~cm}$ above the mesh, which tests showed reduced the attractive force to below the resolution limit of the balance. Figure 5 shows the balance and stand used in these measurements.

In order to make direct comparisons to the stagnation probe measurements, the balance data must be converted into a force per unit length. We do this by dividing the total measured force by the width of buried or wide electrode $(6 \mathrm{~cm})$, which approximates the plasma width. We estimate the plasma width variation from actuator to actuator to be on the order of $\pm 5 \%$, leading to an error the same order as the uncertainty in the force measurement itself.

\section{Power measurements and conditioning}

To determine the electrical power dissipated by the plasma actuators, we placed a $0.10 \mu \mathrm{F}$ capacitor in the ground path of the actuator and measured the voltage across it. Provided the measurement capacitance is much larger than the capacitance of the actuators (which were generally smaller than $10 \mathrm{pF}$ ), the circuit 


\section{AIAA-2009-485 47th ASM, Orlando, FL}

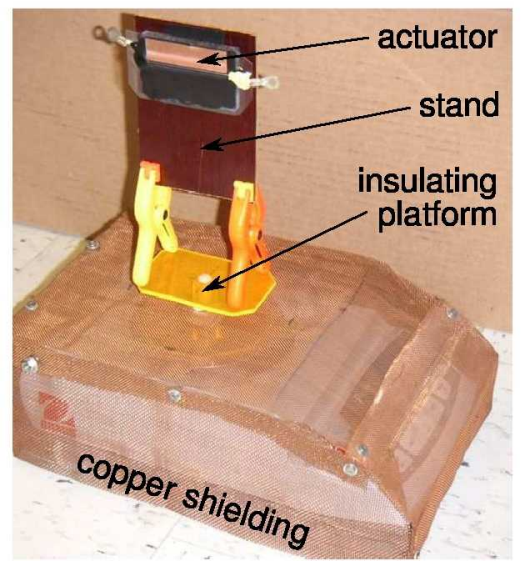

Figure 5. An actuator, actuator stand, insulating platform, and shielded balance used in direct force measurements

characteristics are unaffected by its presence. The actuator current is found by taking the time derivative of the charge on the capacitor, and the time-averaged power determined by integrating the product of the applied voltage and the actuator current over an integer number of voltage cycles. ${ }^{9}$

Previous measurements have shown that actuator behavior exhibits run-to-run variation unless the discharge is operated for some time immediately prior to measurement. ${ }^{14,15}$ To ensure repeatability, all measurements presented here were recorded immediately after the actuators were run at the specified voltage level for at least $30 \mathrm{~min}$.

\section{Results and Discussion}

\section{A. Single Barrier Actuator Measurements}

We have conducted force measurements on single barrier actuators with narrow electrodes ranging from $0.03 \mathrm{~mm}$ to $1.0 \mathrm{~mm}$ in diameter, made from copper $(\mathrm{Cu})$, tungsten (W), or stainless steel (SS). Figure 6 shows the forces per length inferred from these measurements, obtained using the method described in section II.B. We note that at each voltage level, the data are well fit by a simple exponential that decays with increasing electrode diameter,

$$
f=A \exp (-d / L) .
$$

$A$ and $L$ are free parameters, while $d$ is the electrode diameter. The fitted values of $L$ were $0.10,0.11$, and $0.16 \mathrm{~mm}$ for the 6,7 , and $8 \mathrm{kV}$ amplitude tests, respectively. The data also indicate that the material of the exposed electrode plays no role in determining the induced force. The induced forces vary approximately linearly with the amplitude of the applied voltage waveform.

We have also used the electronic balance method described in section II.C to directly measure the induced force of the actuators. The results of these experiments are shown in figure 7 for the same amplitudes of the applied voltage used in the stagnation probe experiments. The balance measurements confirm the two major conclusions of the stagnation probe tests: the exponential dependence of the induced force on the diameter of the exposed electrode, and the independence of the force from the material of the exposed electrode.

It is likely that the major difference between the electrode materials is their differing secondary electron emission coefficients, although the literature regarding these for appropriate ions and energies is sparse. Based on simulation results, Boeuf et al. ${ }^{18}$ predicted that the value of the secondary electron emission coefficient of the electrode (which, in their model, was set equal to the coefficient for the dielectric) would have little effect on the force induced by the actuator, while being a strong predictor of dissipated power. Our data suggests that changing the secondary electron emission coefficient of $j u s t$ the exposed electrode has little effect on the induced force or on the dissipated power (see figure 8).

The electrical power used by each actuator has been measured using the method described in section II.D. The values of this power, shown in figure 8 , do not vary strongly with the exposed electrode diameter, decreasing slightly for actuators with electrode diameters below $0.2 \mathrm{~mm}$. The power varies approximately 


\section{AIAA-2009-485 47th ASM, Orlando, FL}

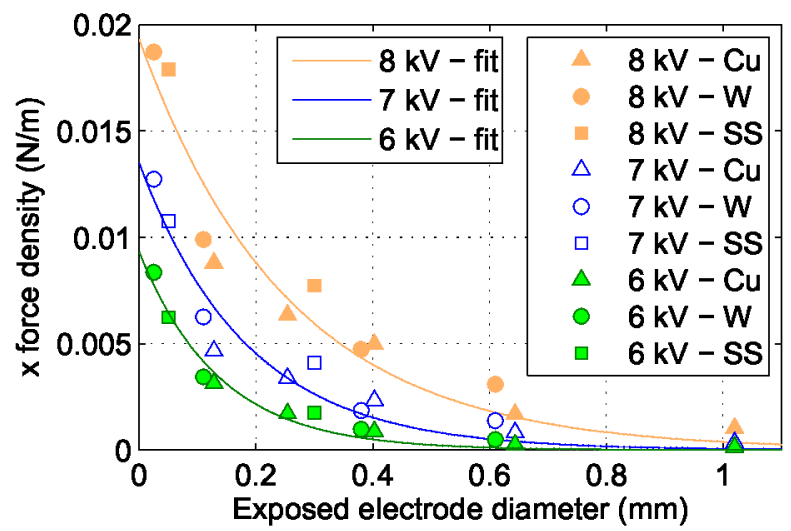

Figure 6. Time-averaged single barrier actuator-induced forces at three different voltage amplitudes, derived from stagnation probe measurements. The exposed electrodes of each actuator were composed of the indicated materials.

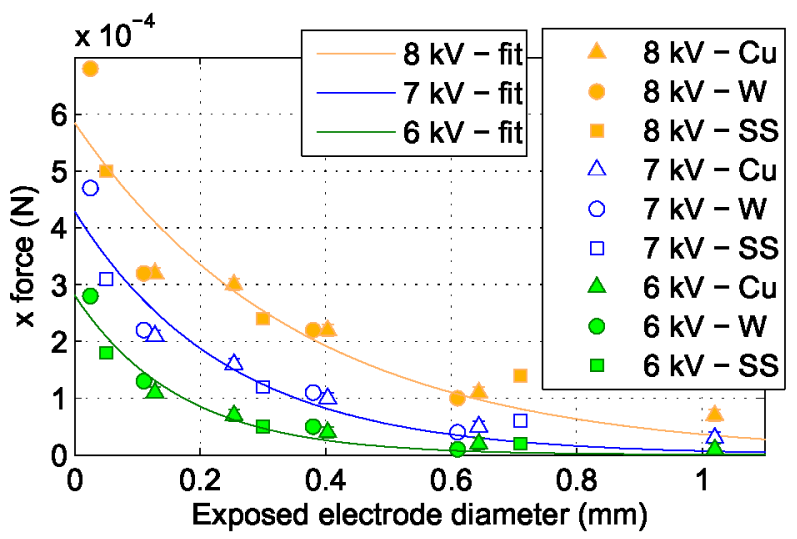

Figure 7. Time-averaged single barrier actuator-induced forces measured using the electronic balance, fitted by exponential decay functions. The exposed electrode of each actuator is composed of the indicated material.

linearly with the amplitude of the applied voltage waveform.

A previous study by Enloe et al. ${ }^{4}$ examined exposed electrode thicknesses down to approximately $0.1 \mathrm{~mm}$, with the smallest electrodes being rectangular rather than cylindrical, and noted a linear trend of increasing force efficiency with decreasing electrode thickness. Our measurements, in contrast, have shown an exponential increase in the force efficiencies as the electrode thickness is reduced. To examine this discrepancy, we constructed and tested several actuators with rectangular cross-section exposed electrodes. These stainlesssteel electrodes were $10 \mathrm{~mm}$ long in the $x$ direction. The actuators had the same $1 \mathrm{~mm}$ horizontal spacing between electrodes as did those using cylindrical electrodes. The results of those measurements along with the data from figure 7 are shown in figure 9 . At present, only actuators with rectangular electrodes of 0.0125 and $0.025 \mathrm{~mm}$ thicknesses have been tested.

The actuators with thin rectangular exposed electrodes induced forces significantly lower than those with cylindrical exposed electrodes of the same thickness. This effect likely explains the discrepancy between our results and those of Enloe et al. ${ }^{4}$ In our tests, the power dissipated by the actuators with rectangular electrodes was about twice as high as the equivalent actuators with cylindrical electrodes. Further testing is necessary to determine the range over which these differences between electrode geometries exist.

\section{B. Double Barrier Measurements}

We have conducted the same force measurements on a series of double barrier actuators, described by figure 2 . Similar to the behavior of single barrier actuators, the double barrier actuators induce larger forces when the electrode diameter, in this case of the narrow electrode, is reduced. Figure 10 shows the forces measured 


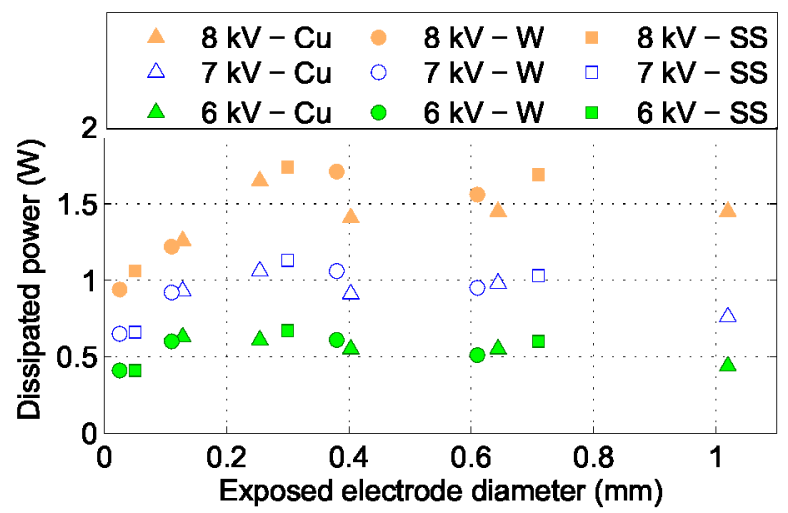

Figure 8. Time-averaged electrical power dissipated by the single barrier actuators

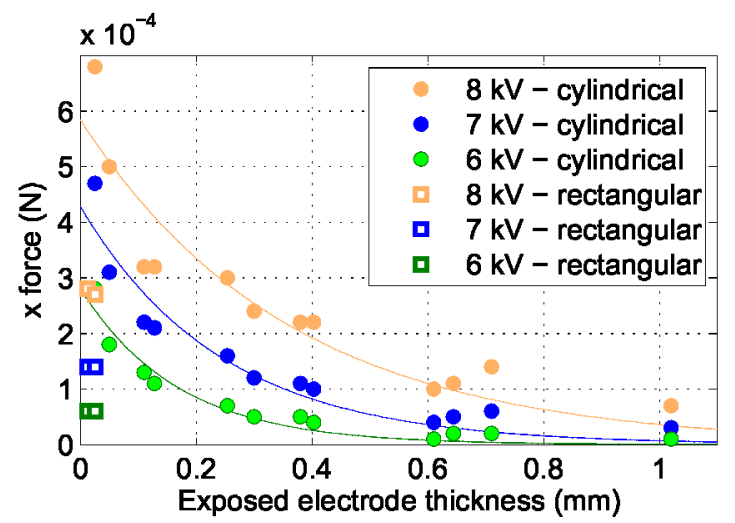

Figure 9. Time-averaged forces induced by single barrier actuators with rectangular and cylindrical exposed electrodes, measured using the electronic balance

using the stagnation probe technique. The measured forces are smaller than those seen for single barrier actuators by roughly a factor of 5 . Electrodes smaller than $0.1 \mathrm{~mm}$ in diameter tended to cause arcing through the dielectric layer, and so could not be studied.

In contrast to the behavior of single barrier actuators, at large diameters the induced force does not decrease to zero but rather asymptotes to a small but finite value. Therefore equation (5) cannot be used to fit the existing data. Instead we use a similar expression, but with an offset term:

$$
f=A \exp (-d / L)+C,
$$

where $A, L$, and $C$ are free parameters. For the fits shown in figure 10, the values of $L$ were $0.26,0.27$, and $0.24 \mathrm{~mm}$ for the 11,12 , and $13 \mathrm{kV}$ tests, respectively. We note that at larger values of narrow electrode diameter, the system geometry does not become symmetric. Rather, the dominant asymmetry may be between the differently-shaped electrode cross sections (circular for the narrow electrode and rectangular for the wide electrode), rather than between their widths.

The double-barrier actuator forces, measured using the electronic balance, are shown in figure 11 along with fits using equation (6). The behavior of the data generally confirms the measurements using the stagnation probe, although the measured forces are small enough to approach the sensitivity limit of the balance. Fitted values of $L$ were similar to those found from the stagnation probe data, at $0.24,0.26$, and $0.29 \mathrm{~mm}$.

In order to produce plasma, double barrier actuators generally require higher applied voltages when compared to single barrier actuators. The rates of discharge events, and therefore the electrical current, are much lower. This combination leads to slightly lower power use by double barrier actuators in the parameter ranges tested here. The measured powers are shown in figure 12. As with single barrier actuators, the power 


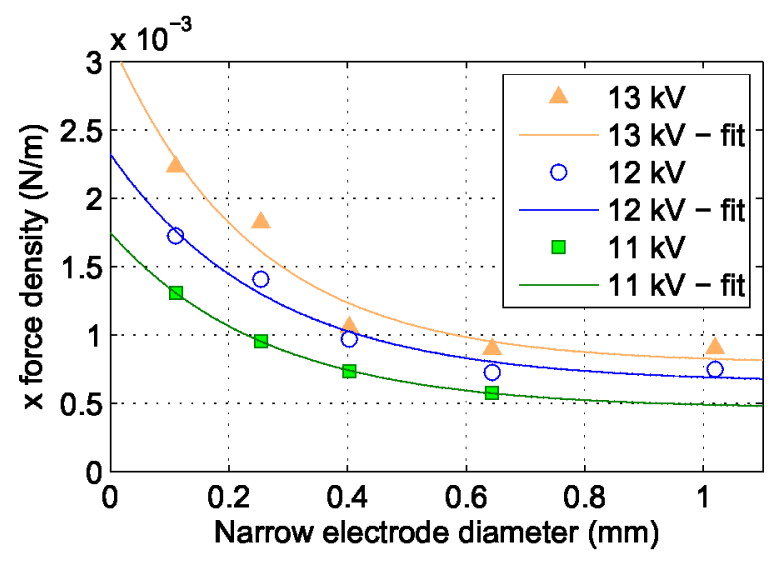

Figure 10. Time-averaged double barrier actuator-induced forces derived from stagnation probe measurements

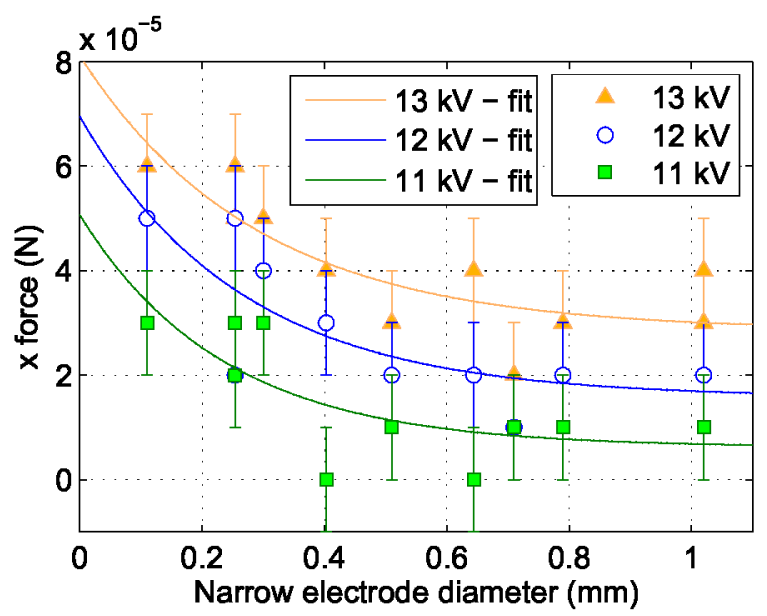

Figure 11. Time-averaged double barrier actuator-induced forces measured using the electronic balance, along with analytic fits to the data.

decreases slightly at smaller electrode diameters, meaning the increased force seen for those electrodes is not due to increased power dissipation, but is rather increased efficiency.

\section{Comparison of Measurement Methods}

Nearly identical trends in the induced force with varying electrode diameter have been observed using both a stagnation probe technique and an electronic balance. When the force densities are quantitatively compared, as in figure 13, the balance data are consistently lower. While the two approaches are clearly proportional to each other, the stagnation probe data are approximately a factor of two larger for all actuators.

We have determined that the differences between the two techniques are largely due to the assumptions made in section II.B to convert the measured stagnation pressures into force densities. To show this, we have made additional detailed measurements around one actuator (a single barrier actuator with a $0.11 \mathrm{~mm}$ diameter exposed electrode, driven by a $7 \mathrm{kV}$ amplitude triangle wave), and found that the corrections provided by these data are sufficient to explain this observed difference between stagnation probe and balance data.

We measured variations in both stagnation and static pressures at several $x$ locations, as shown in figure 14. Static pressures were measured using a $2.5 \mathrm{~mm}$ diameter alumina tube sealed at one end, with a $1 \mathrm{~mm}$ diameter hole drilled in the side $7 \mathrm{~mm}$ from the sealed end. The tube was oriented in the $-x$ direction, with the hole facing the $z$ direction. Stagnation and static pressure measurements at three locations are shown in figure 14. 


\section{AIAA-2009-485 47th ASM, Orlando, FL}

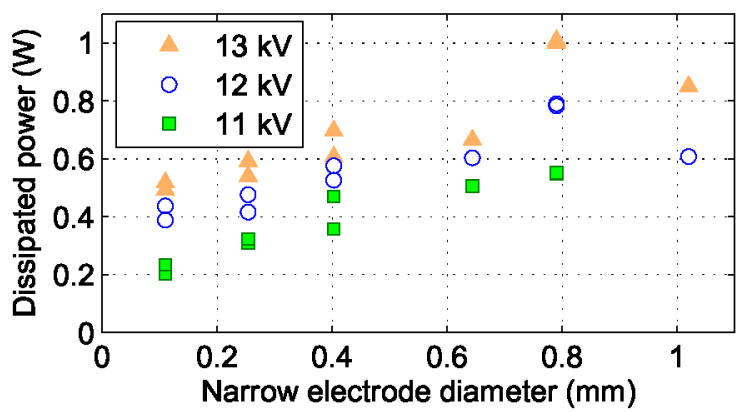

Figure 12. Time-averaged electrical power dissipated by double barrier actuators. Some actuators have been tested multiple times.

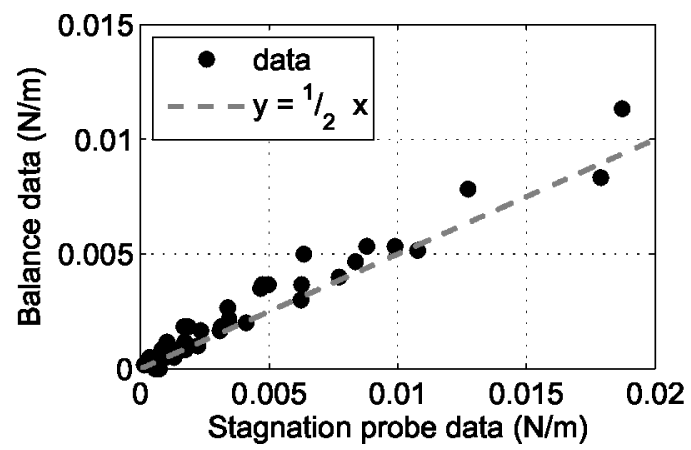

Figure 13. Comparison of force densities measured using the electronic balance and calculated from the stagnation probe data using the assumptions of section II.B

The measurements confirm that the static pressure is constant downstream ( $+x$ direction) of the high voltage electrode, but show a small non-zero static pressure upstream. Using these data we can evaluate the right-hand side of equation (1), except for term II, using the measured static pressure in place of the reference pressure in equation (3). Evaluating terms I and IV along the surface at $x=8 \mathrm{~mm}$ and terms III and $\mathrm{V}$ on the $x=-4 \mathrm{~mm}$ surface, we find a linear force density of $4.2 \times 10^{-3} \mathrm{~N} / \mathrm{m}$, or $68 \%$ of the original force estimate for these conditions. If we repeat the calculation and instead evaluate terms I and IV at $x=16 \mathrm{~mm}$, thereby including the shear force all the way to the actuator edge, we calculate a linear force density of $3.3 \times 10^{-3} \mathrm{~N} / \mathrm{m}$. This is $53 \%$ of the original estimate, and compares well to the balance-measured value of $3.7 \times 10^{-3} \mathrm{~N} / \mathrm{m}$.

The significantly improved agreement between the two measurement techniques indicates that the assumptions made in section II.B are not strictly valid. The largest correction to our original force estimate was caused by term III in equation (1), the flux of momentum into the left-hand side of the control volume, which was equal to $35 \%$ of the uncorrected force. Our estimate of term III in equation (1) itself may be slightly in error: the static probe was large enough to possibly disturb the gas flow in the region of measurement. Still, we believe including the term as measured is likely to be more accurate than continuing to neglect it completely.

We have not estimated the effect of the flux of $x$ momentum through the top surface (term II in equation (1)), as we cannot accurately measure $v_{y}$ in that region. PIV measurements and calculations ${ }^{19,20}$ suggest that on the top surface $v_{x}$ is much smaller than its values on the left- and right-hand surfaces of the control volume and comparable to $v_{y}$, because most of the acceleration occurs inside the control volume closer to the surface. This behavior would make our neglect of term II reasonable. The relatively close agreement between the corrected stagnation probe and balance measurements also supports this conclusion. 


\section{AIAA-2009-485 47th ASM, Orlando, FL}
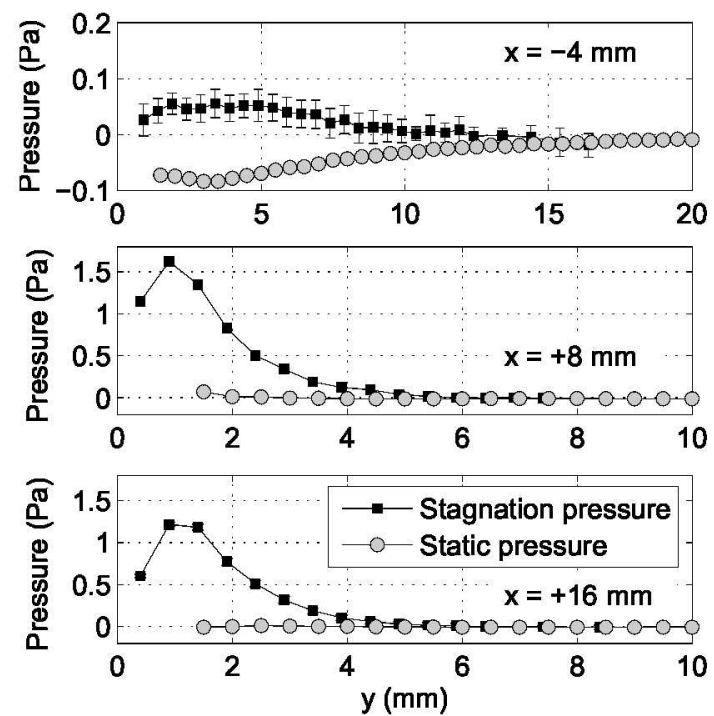

Figure 14. Stagnation and static pressures measured at $x=-4$ (top), +8 (middle), and $+16 \mathrm{~mm}$ (bottom) for a single barrier actuator with $d=0.11 \mathrm{~m}$ driven at $7 \mathrm{kV}$

\section{Conclusion}

We have observed exponential increases in the force induced by single barrier actuators as the exposed electrode diameter decreases. These forces have been shown to be independent of the material from which the exposed electrode is constructed. These behaviors have been confirmed using two independent techniques: direct measurement using an electronic balance and calculating the force from stagnation probe measurements. Simplifying assumptions used in calculating the induced forces from stagnation probe measurements tended to cause overestimations of the force. Reducing the actuator diameter to the extent possible suggests a simple way to significantly increase the force induced by these actuators. The same two measurement techniques have shown the forces induced by double barrier actuators to increase as the narrow electrode diameter is reduced.

\section{Acknowledgments}

This work was supported by NASA under cooperative agreement NNX07AB84A and the Air Force Office of Scientific Research (AFOSR) under grant FA9550-07-1-0025. The authors thank Dr. Riccardo Bonazza for helpful discussions regarding the fluid dynamics calculations, and Dr. Jon Ahn for suggestions regarding our pressure measurement set-up.

\section{References}

${ }^{1}$ Roth, J. R., Sherman, D. M., and Wilkinsons, S. P., "Electrohydrodynamic flow control with a Glow-Discharge Surface Plasma," AIAA J., Vol. 38, No. 7, July 2000, pp. 1166-1172.

${ }^{2}$ Hultgren, L. S. and Ashpis, D. E., "Demonstration of separation delay with glow-discharge plasma actuators," 41st AIAA Aerospace Sciences Meeting, Jan. 2003.

${ }^{3}$ Post, M. L. and Corke, T. C., "Separation control on high angle of attack airfoil using plasma actuators," AIAA J., Vol. 42, No. 11, Nov. 2004, pp. 2177-2184.

${ }^{4}$ Enloe, C. L., McLaughlin, T. E., VanDyken, R. D., Kachner, K. D., Jumper, E. J., Corke, T. C., Post, M., and Haddad, O., "Mechanisms and responses of a single dielectric barrier plasma actuator: Geometric effects," AIAA J., Vol. 42, No. 3, Mar. 2004, pp. 595-604.

${ }^{5}$ Opaits, D. F., Neretti, G., V., L. A., Zaidi, S., Shneider, M. N., and Miles, R. B., "Experimental investigation of DBD plasma actuators driven by repetitive high voltage nanosecond pulses with DC or low-frequency sinusoidal bias," 38 th $A I A A$ Plasmadynamics and Lasers Conference, June 2007.

${ }^{6}$ Boeuf, J. P. and Pitchford, L. C., "Electrohydrodynamic force and aerodynamic flow acceleration in surface dielectric barrier discharge," J. Appl. Phys., Vol. 97, No. 103307, May 2005. 


\section{AIAA-2009-485 47th ASM, Orlando, FL}

${ }^{7}$ Roth, J. R., "Aerodynamic flow acceleration using paraelectric and peristaltic electrohydrodynamic effects of a One Atmosphere Uniform Glow Discharge Plasma," Phys. Plasmas, Vol. 10, No. 5, May 2003, pp. 2117-2126.

${ }^{8}$ Enloe, C. L., McLaughlin, T. E., VanDyken, R. D., Kachner, K. D., Jumper, E. J., and Corke, T. C., "Mechanisms and responses of a single dielectric barrier plasma actuator: Plasma morphology," AIAA J., Vol. 42, No. 3, 2004, pp. 589-594.

${ }^{9}$ Pons, J., Moreau, E., and Touchard, G., "Asymmetric surface dielectric barrier discharge in air at atmospheric pressure: Electrical properties and induced airflow characteristics," J. Phys. D, Vol. 38, Sep. 2005, pp. 3635-3642.

${ }^{10}$ Enloe, C. L., McLaughlin, T. E., Gregory, J. W., Medina, R. A., and Miller, W. S., "Surface Potential and Electric Field Structure in the Aerodynamic Plasma Actuator," 46th AIA A Aerospace Sciences Meeting, Jan. 2008.

${ }^{11}$ Likhanskii, A. V., Shneider, M. N., Macheret, S. O., and Miles, R. B., "Modeling of interaction between weakly ionized near-surface plasmas and gas flow," 44th AIAA Aerospace Sciences Meeting, Jan. 2006.

${ }^{12}$ Boeuf, J. P., Lagmich, Y., Unfer, T., Callegari, T., and Pitchford, L. C., "Electrohydrodynamic force in dielectric barrier discharge plasma actuators," J. Phys. D, Vol. 40, Jan. 2007, pp. 652-662.

${ }^{13}$ Likhanskii, A. V., Shneider, M. N., Macheret, S. O., and Miles, R. B., "Modeling of dielectric barrier discharge plasma actuators driven by repetitive nanosecond pulses," Phys. Plasmas, Vol. 14, No. 073501, July 2007.

${ }^{14}$ Hoskinson, A. R. and Hershkowitz, N., "Flow measurements and plasma simulations of double and single barrier DBD plasma actuators in quiescent air," 46th AIAA Aerospace Sciences Meeting, Jan. 2008, pp. 2008-1370.

${ }^{15}$ Hoskinson, A. R., Hershkowitz, N., and Ashpis, D. E., "Force measurements of single and double barrier DBD plasma actuators in quiescent air," J. Phys. D, Vol. 41, Nov. 2008, pp. 245209.

${ }^{16}$ Baughn, J. W., Portoer, C. O., Peterson, B. L., McLaughlin, T. E., Enloe, C. L., Font, G. I., and Baird, C., "Momentum Transfer for an Aerodynamic Plasma Actuator with an Imposed Boundary Layer," 44th AIAA Aerospace Sciences Meeting, Jan. 2006.

${ }^{17}$ Enloe, C. L., McLaughlin, T. E., and Font, G. I., "Parameterization of temporal structure in the single-dielectric-barrier aerodynamic plasma actuator," AIAA J., Vol. 44, No. 6, June 2006, pp. 1127-1136.

${ }^{18}$ Boeuf, J. P., Lagmich, Y., Callegari, T., Pitchford, L. C., and Unfer, T., "New Insights in the Physics of DBD Plasma Actuators for Flow Control," 46th AIAA Aerospace Sciences Meeting, Jan. 2008.

${ }^{19}$ Suzen, Y. B., Huang, P. G., Jacob, J. D., and Ashpis, D. E., "Numerical simulations of plasma based flow control applications," 35th Fluid Dynamics Conference, June 2005.

${ }^{20}$ Santhanakrishnan, A., Jacob, J. D., and Suzen, Y. B., "Flow Control Using Plasma Actuators and Linear/Annular Plasma Synthetic Jet Actuators," 3rd AIAA Flow Control Conference, June 2006. 